Original Research Paper

\title{
Mycobacterium Chimaera. A Lethal Enemy of Cardiac Surgery
}

\author{
${ }^{1}$ Anna Llorens Ferrer, ${ }^{1}$ Elisabet Berastegui Garcia, ${ }^{2}$ Lourdes Mateu Pruñosa and ${ }^{1}$ Christian Muñoz Guijosa \\ ${ }^{1}$ Department of Cardiac Surgery, Hospital Universitari Germans Trias i Pujol, Crtra Canyet s/n, 08914, Badalona, Spain \\ ${ }^{2}$ Department of Infectious Disease, Hospital Universitari Germans Trias i Pujol, Crtra Canyet s/n, 08914, Badalona, Spain
}

\author{
Article history \\ Received: 02-03-2021 \\ Revised: 22-04-2021 \\ Accepted: 28-04-2021 \\ Corresponding Author: \\ Anna Llorens Ferrer \\ Department of Cardiac Surgery, \\ Hospital Universitari Germans \\ Trias i Pujol, Crtra Canyet s/n, \\ 08914, Badalona, Spain \\ Email: allorens.germanstrias@gencat.cat
}

\begin{abstract}
Mycobacterium chimaera (M. chimaera) can cause disseminated infection and Infective Endocarditis (IE) in patients with previous cardiac surgery. M. chimaera is acquired during cardiopulmonary bypass via bioaerosols emitted from contaminated heater-cooler units water systems. M. chimaera infections can cause pneumonia, especially in patients with underlying chronic pulmonary diseases like cystic fibrosis and Chronic Obstructive Pulmonary Disease (COPD), cardiovascular manifestations and disseminated mycobacterial infection. The prognosis is extremely poor and early diagnosis and treatment are decisive to avoid fatality. Nevertheless, an early diagnosis is difficult due to intermittent bacteremia and normal echocardiography and it requires a high index of suspicion. An early use of FDG PET/CT could improve an accurate diagnosis and reduce morbidity and mortality. We report an illustrative case of a $M$. chimaera IE with disseminated infection in a patient suffering from COPD who underwent heart surgery.
\end{abstract}

Keywords: Mycobacterium Chimaera, Infective Endocarditis

\section{Introduction}

Mycobacterium chimaera (M. chimaera) is a nontuberculous mycobacterium of the Mycobacterium avium complex (Tortoli et al., 2004). It was first described in 2004 as a cause of respiratory infection in patients with Chronic Obstructive Pulmonary Disease (COPD) and disseminated infection in immunocompromised patients (Bills et al., 2009). In 2011, it was associated with disseminated infection and Infective Endocarditis (IE) in patients with previous history of cardiovascular surgery with Extracorporeal Circulation (EC) (Achermann et al., 2013). Heater-cooler units are an essential part of EC because they help keep a patient's circulating blood at a specific temperature during the procedure. Some LivaNova 3T heater-cooler devices (Livanova, Saluggia, Italy), used during many open-heart surgeries, might have been contaminated during manufacturing, which could put patients at risk for the infection (Haller et al., 2016; van Ingen et al., 2017). Although water from the device is not intended to contact the patient directly, given its capacity to form biofilms and under certain circumstances, due to fluid leakage and/or aerosolization, M. chimaera could reach a patient's surgical site (Sommerstein et al., 2016). Clinical manifestations can take months to appear after the cardiac surgery and they usually present as unspecific symptoms such as weight loss, asthenia, fever or night sweats (Scriven et al., 2018; Marra et al., 2017). The delay in diagnosis and treatment has an impact on morbidity and mortality due to systemic involvement.

We present a case of disseminated disease and IE due to $M$. chimaera in a patient with prior aortic valve replacement with a sutureless Perceval S (Livanova, Saluggia, Italy) valve. This new prosthesis is formed by three sheets of bovine pericardium, mounted on a flexible external nitinol stent. Its use has been rapidly widespread thanks to its ease of implantation and its favourable hemodynamic profile. Its special design produces that clinical and echocardiographic presentation of IE in this type of prosthesis has some special characteristics that are also manifested in this report.

\section{Case Presentation}

It is a 72-year-old male patient, with previous COPD, Obstructive Sleep Apnea Syndrome (OSAS), pulmonary hypertension and mild right ventricular dysfunction. Due to symptomatic severe aortic stenosis and significant disease of the Left Anterior Descending (LAD) coronary artery, in July 2016 underwent aortic valve replacement with a Perceval S bioprosthesis and left internal mammary artery to LAD coronary bypass graft. Ten days after surgery, a DDD pacemaker was implanted due to complete atrioventricular block (Fig. 1). 
In December 2016, the patient was hospitalized for prolonged fever with shivering, headache and abdominal discomfort. At physical examination, a systolic murmur and hepatomegaly were observed and laboratory tests showed increased C-Reactive Protein (CRP). Urine, sputum and blood cultures were taken and empiric antibiotics with vancomycin and gentamicin were administered on suspicion of IE. A Transthoracic (TTE) and Transesophageal (TEE) echocardiogram showed a normally functioning aortic bioprosthesis without images of vegetations or periannular complications. However, due to clinical suspicion of IE, a [18F]-Fluorodeoxyglucose Positron Emission Tomography combined with Computed Tomography (FDG PET/CT) was performed. Focal fixation of FDG in the annulus of the aortic bioprosthesis was observed, mainly below the right coronary sinus, without evidence of pacemaker infection (Fig. 2a). The patient presented hepatomegaly associated with pancytopenia and after an inconclusive bone marrow biopsy and a normal fibrogastroscopy, a liver biopsy was performed. Results demonstrated a granulomatous hepatitis suggestive of tuberculosis or sarcoidosis. Based on these findings, empirical treatment for tuberculosis was initiated with clinical and analytic improvement. Two weeks later, $M$. chimaera was identified in urine, sputum and blood cultures, using Löwenstein-Jensen agar Medium. The patient was discharged home with rifampicin $(600 \mathrm{mg}$ orally once daily), clarithromycin (500 mg orally twice daily) and ethambutol $(800 \mathrm{mg}$ orally twice daily) with the diagnosis of disseminated disease and IE due to M. chimaera.
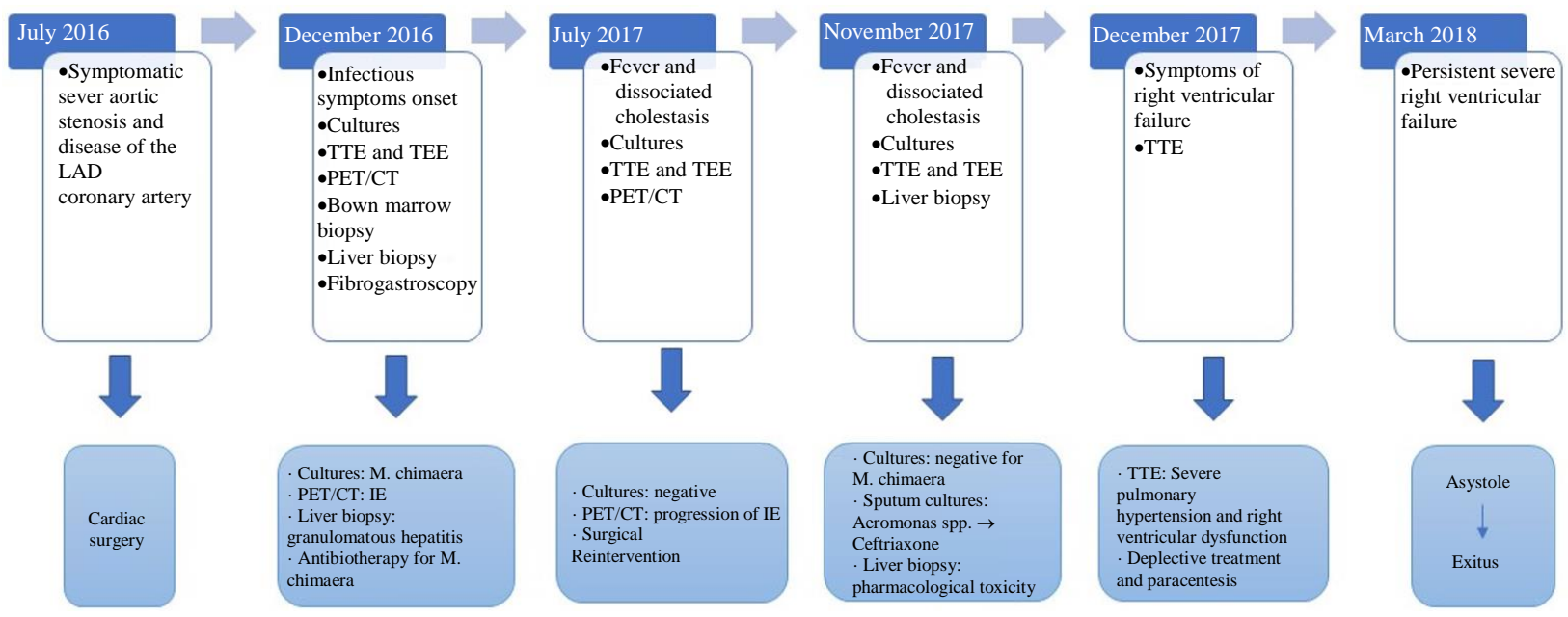

Fig. 1: It summarizes the sequence of medical events, diagnostic methods and therapeutic actions

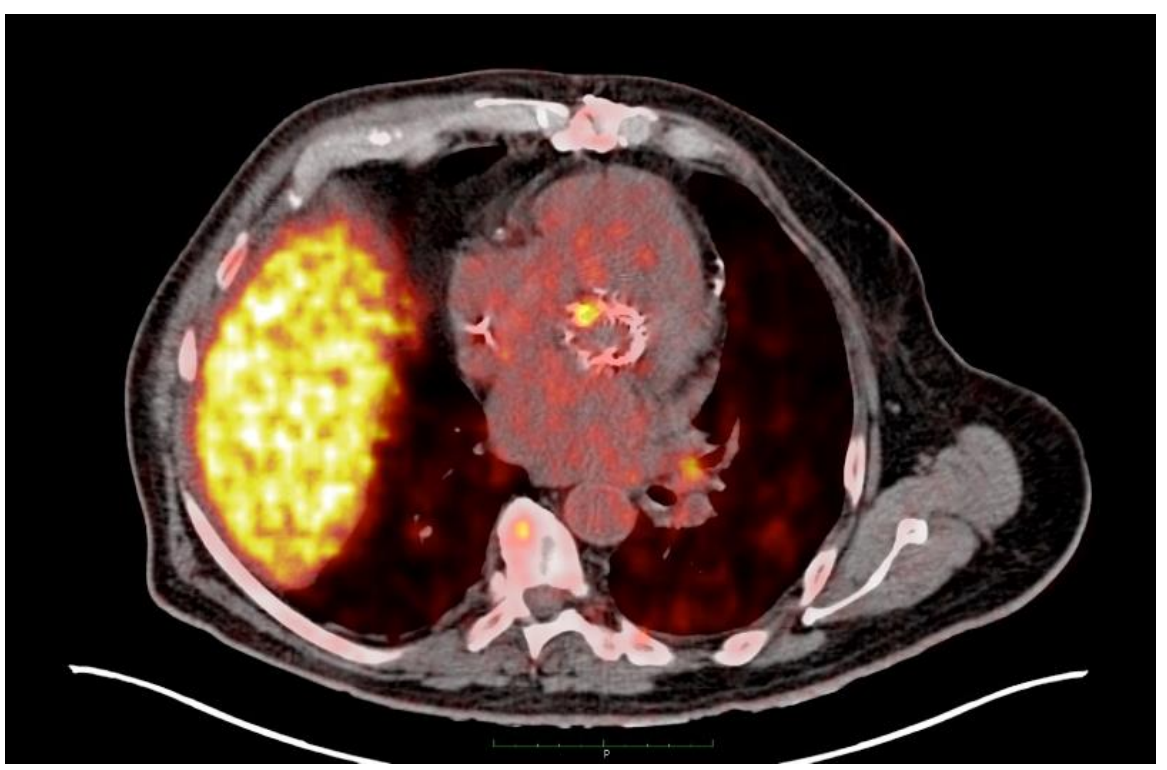

(a) 


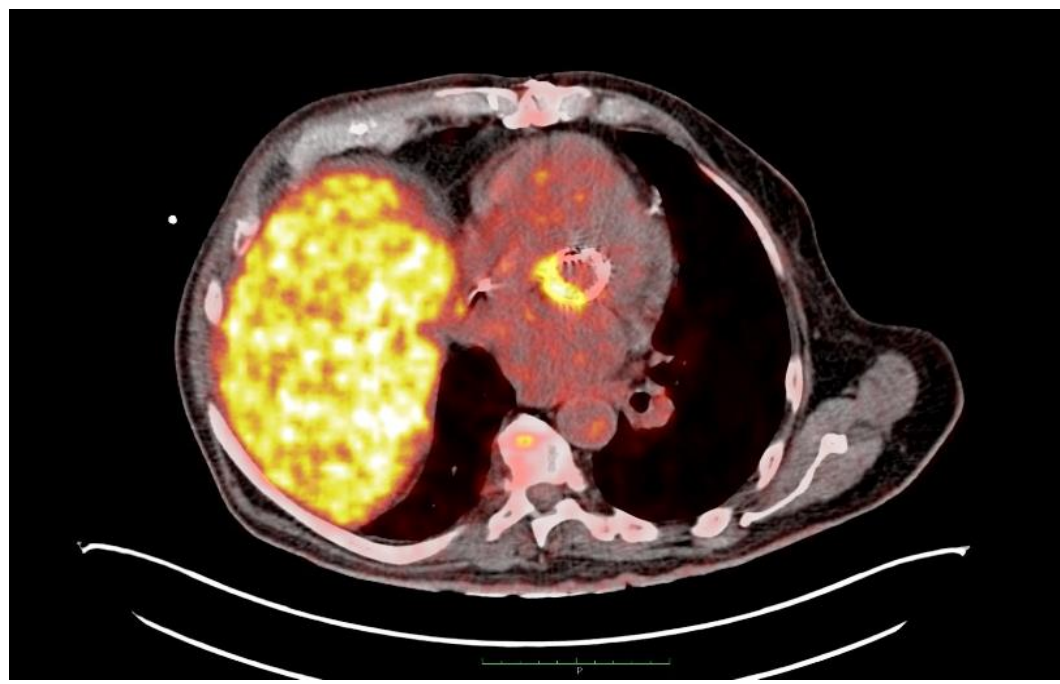

(b)

Fig. 2: (a) An early PET/CT showed focal fixation of FDG in the annulus of the aortic bioprosthesis, mainly bellow the right coronary sinus (b) A posterior PET/CT confirmed morph metabolic progression with respect to the previous study, with fixation of FDG in the posterior annulus in continuity with anterior mitral valve leaflet, compatible with periaortic abscess

In July 2017, the patient was re-hospitalized because of febrile syndrome and dissociated cholestasis. A TEE showed an heterogenous image at the posterior annulus in continuity with anterior mitral valve leaflet, compatible with periaortic abscess. A new FDG PET/CT scan was performed and confirmed morphologic and metabolic progression with respect to the previous study, without evidence of pacemaker infection (Image 1b). Decision for surgical re-intervention was made. Aortic bioprosthesis and pacemaker were both removed and a new Perceval S prosthesis and an epicardial electrocatheter were implanted. Prosthetic valve cultures were negative, as well as blood cultures perfomed during hospitalization. The patient was discharged home with clarithromycin (500 mg orally once daily), clofazimine (100 mg orally once daily) and ethambutol (400 mg orally three times daily).

In November 2017, the patient was hospitalized because of fever and alteration of liver biochemistry. Another liver biopsy was indicated. Histopathological study revealed acute granulomatous hepatitis with hepatocyte ballooning, findings consistent with both toxicity and infection. Cultures were negative for M. chimaera, which suggests that liver alteration was in part of pharmacological cause. A sputum culture was positive for Aeromonas spp., so ceftriaxone was added in the treatment.

Two months later, the patient was again re-admitted to hospital due to NYHA III functional class, orthopnea, edema and abdominal distention. A TTE confirmed severe right ventricular dysfunction, severe tricuspid regurgitation and severe pulmonary hypertension and an abdominal echography, abundant free intra-abdominal fluid. Despite intense depletive treatment and repeated paracentesis, the patient persisted with edema and ascites and finally he suffered a cardiorespiratory arrest due to asystole, from which, unfortunately, he could not recover.

\section{Discussion}

This case reflects the diagnostic difficulty of systemic infections caused by $M$. chimaera. It results from various factors: Symptoms may take months or years to appear after extracorporeal surgery, clinical presentation is almost always nonspecific and of subacute onset, physical examination does not show specific findings, bacteremia is usually intermittent and there is a low yield of tissue culture positivity early in the infection (Kasperbauer and Daley, 2019). Furthermore $M$. chimaera is slow growing and even with specific culture methods, detection may take between 2 and 8 weeks. Given that symptoms of infection can take a long time to develop and they are often general and nonspecific, M. chimaera infection is not usually considered as the first possibility, which causes that its diagnostic can be delayed, making these infections more difficult to treat. Delayed diagnosis can result in more widespread disease. Its suspicion is mandatory in a patient with history of surgery requiring cardiopulmonary bypass in the five years prior to the onset of symptoms of infection and any of the following clinical criteria: Prosthetic valve endocarditis, prosthetic vascular graft infection, sternotomy wound infection, mediastinitis or manifestation of disseminated infection including embolic and immunologic manifestations, e.g. splenomegaly, arthritis, osteomyelitis, bone marrow involvement with cytopenia, chorioretinitis, lung involvement, hepatitis, nephritis or myocarditis (Casalta, 2015; Hasse et al., 2020). 
In relation to diagnostic imaging tests, TTE or TEE diagnostic criteria in prosthetic IE have shown low sensitivity. Therefore, in case of possible IE according to traditional criteria but with high clinical suspicion, the latest guidelines recommend the use of FDG PET/CT in the diagnosis. Furthermore, this technique gives us information about possible complications of IE, such as perivalvular extension or systemic embolism. It can also be employed to monitor response to antimicrobial treatment in patients with established IE (Habib et al., 2015).

On the other hand, this patient presented a prosthetic IE on a sutureless Perceval S prosthesis. Its clinical and echocardiographic presentation is characterised by the absence of structural dysfunction and periprosthetic leak and the highest frequency of periannular complications. It could be due to the radial force exerted by the nitinol stent, which keeps the prosthetic ring in close contact with the native valve ring. This specific presentation form also makes the echocardiographic diagnosis of IE on Perceval prosthesis more difficult, so in this scenario, FDG PET/CT can be the definitive diagnostic tool.

Regarding to the treatment of $M$. chimaera disease, the use of clarithromycin or azithromycin, rifabutin or rifampicin and ethambutol, with the possibility of adding an intravenous aminoglycoside (amikacin) or a fluoroquinolone (moxifloxacin) are currently the preferred combinations (Campins et al., 2019). Due to the capacity of $M$. chimaera to construct biofilms, as well as the difficulty to negativise cultures despite prolonged antibiotic treatment, an aggressive and early surgical intervention removing and replacing prosthetic material is of great importance and may represent the only option of cure in these patients (Scriven et al., 2018).

Future areas of research should be focused on device safety and collaborative discussions between medical device manufacturers, engineers and hospital epidemiology experts should be needed as new heatercooler units are designed (Hasse et al., 2020). On the other hand, we think that multicentre registers providing data on epidemiology, clinical manifestations, treatment and outcomes would help to direct diagnosis and management of $M$. Chimaera infections.

\section{Conclusion}

IE and disseminated infection due to $M$. chimaera in patients with previous cardiac surgery have a high morbidity and mortality. Regardless our limited experience with a single case, we want to highlight the importance of an early diagnosis to obtain better clinical results. To avoid delays in the diagnosis and treatment of the disease, an early FDG PET/CT may be performed, given that echocardiographic findings have low sensibility. A combined approach with antibiotherapy and early and aggressive surgical removal of all prosthetic matherial is essential to reduce fatality.

\section{Funding}

Authors received no financial support for the research, authorship and publication of this article.

\section{Author's Contributions}

Anna Llorens Ferrer: Design and writing of the article.

Elisabet Berastegui Garcia and Christian Muñoz Guijosa: Critical review of the article for significant intellectual content.

Lourdes Mateu Pruñosa: Data acquisition and analysis and interpretation of data.

\section{Ethics Approval and Consent to Participate}

This article is original and contains unpublished material. The corresponding author confirms that all of the other authors have read and approved the manuscript and no ethical issues involved.

\section{References}

Achermann, Y., Rössle, M., Hoffmann, M., Deggim, V., Kuster, S., Zimmermann, D. R., ... \& Hasse, B. (2013). Prosthetic valve endocarditis and bloodstream infection due to Mycobacterium chimaera. Journal of Clinical Microbiology, 51(6), 1769-1773.

https://jcm.asm.org/content/51/6/1769.short

Bills, N. D., Hinrichs, S. H., Aden, T. A., Wickert, R. S., \& Iwen, P. C. (2009). Molecular identification of Mycobacterium chimaera as a cause of infection in a patient with chronic obstructive pulmonary disease. Diagnostic Microbiology and Infectious Disease, 63(3), 292-295. https://doi.org/10.1016/j.diagmicrobio.2008.12.002

Campins, M., Borrás, B., \& Armadans, L. (2019). Mycobacterium chimaera infections and cardiac surgery. An unsolved problem. Clinical Medicine, $152 \quad(8), \quad 317-323$. https://doi.org/10.1016/j.medcli.2018.10.013

Casalta, J. P., Del Zotti, F., Dulgheru, R., El Khoury, G., Erba, P. A., Iung, B., Miro, J. M., Mulder, B. J., PlonskaGosciniak, E., Price, S., Roos-Hesselink, J., SnyggMartin, U., Thuny, F., Tornos Mas, P., Vilacosta, I., Zamorano, J. L., \& Scientific Document Group, E. S. C. (2015). ESC Guidelines for the management of infective endocarditis: The Task Force for the Management of Infective Endocarditis of the European Society of Cardiology (ESC). Endorsed by: European Association for Cardio-Thoracic Surgery (EACTS), the European Association of Nuclear Medicine (EANM). Eur Heart J. 2015 Nov 21; 36(44):3075-3128. https://doi.org/10.1093/eurheartj/ehv319. PMID: 26320109. 
Habib, G., Lancellotti, P., Antunes, M. J., \& Bongiorni, M. G. (2015). European Centre for Disease Prevention and Control. EU. protocol for case detection, laboratory diagnosis and environmental testing of Mycobacterium chimaera infections potentially associated with heater-cooler units: case definition and environmental testing methodology. Stockholm: ECDC; 2015

Haller, S., Höller, C., Jacobshagen, A., Hamouda, O., Sin, M. A., Monnet, D. L., ... \& Eckmanns, T. (2016). Contamination during production of heater-cooler units by Mycobacterium chimaera potential cause for invasive cardiovascular infections: results of an outbreak investigation in Germany, April 2015 to February 2016. Eurosurveillance, $\quad 21(17), \quad 30215$. https://www.eurosurveillance.org/content/10.2807 /1560-7917.ES.2016.21.17.30215?crawler=true

Hasse, B., Hannan, M. M., Keller, P. M., Maurer, F. P., Sommerstein, R., Mertz, D., .. \& ISCVID Executive Committee. (2020). International Society of Cardiovascular Infectious Diseases Guidelines for the diagnosis, treatment and prevention of disseminated Mycobacterium chimaera infection following cardiac surgery with cardiopulmonary bypass. Journal of Hospital Infection, 104(2), 214-235. https://doi.org/10.1016/j.jhin.2019.10.009

Kasperbauer, S. H., \& Daley, C. L. (2019). Mycobacterium chimaera infections related to the heater-cooler unit outbreak: a guide to diagnosis and management. Clinical Infectious Diseases, $68(7)$ 1244-1250. https://academic.oup.com/cid/articleabstract/68/7/1244/5145970
Sommerstein, R., Rüegg, C., Kohler, P., Bloemberg, G., Kuster, S. P., \& Sax, H. (2016). Transmission of Mycobacterium chimaera from heater-cooler units during cardiac surgery despite an ultraclean air ventilation system. Emerging Infectious Diseases, 22(6), 1008. https://www.ncbi.nlm.nih.gov/pmc/articles/PMC488 0077/

Marra, A. R., Diekema, D. J., \& Edmond, M. B. (2017). Mycobacterium chimaera infections associated with contaminated heater-cooler devices for cardiac surgery: outbreak management. Clinical Infectious Diseases, 65(4), 669-674. https://academic.oup.com/cid/articleabstract/65/4/669/3739869

Scriven, J. E., Scobie, A., Verlander, N. Q., Houston, A., Collyns, T., Cajic, V., ... \& Chand, M. (2018). Mycobacterium chimaera infection following cardiac surgery in the United Kingdom: clinical features and outcome of the first 30 cases. Clinical Microbiology and Infection, 24(11), 1164-1170. https://doi.org/10.1016/j.cmi.2018.04.027

Tortoli, E., Rindi, L., Garcia, M. J., Chiaradonna, P., Dei, R., Garzelli, C., ... \& Scarparo, C. (2004). Proposal to elevate the genetic variant MAC-A, included in the Mycobacterium avium complex, to species rank as Mycobacterium chimaera sp. nov. International Journal of Systematic and Evolutionary Microbiology, 54(4), 1277-1285. https://www.microbiologyresearch.org/content/journ al/ijsem/10.1099/ijs.0.02777-0?crawler=true

Van Ingen, J., Kohl, T. A., Kranzer, K., Hasse, B., Keller, P. M., Szafrańska, A. K., ... \& Sax, H. (2017). Global outbreak of severe Mycobacterium chimaera disease after cardiac surgery: a molecular epidemiological study. The Lancet Infectious Diseases, 17(10), 1033-1041. https://doi.org/10.1016/S1473-3099(17)30324-9. 